Research Paper

\title{
Feasible Advantage of Bioactive/Bioresorbable Devices Made of Forged Composites of Hydroxyapatite Particles and Poly-L-lactide in Alveolar Bone Augmentation: A Preliminary Study
}

\author{
Shintaro Sukegawa ${ }^{\bowtie}$, Hotaka Kawai², Keisuke Nakano², Takahiro Kanno ${ }^{1,3}$, Kiyofumi Takabatake², \\ Hitoshi Nagatsuka², Yoshihiko Furuki ${ }^{1}$ \\ 1. Division of Oral and Maxillofacial Surgery, Kagawa Prefectural Central Hospital, Takamatsu, Japan \\ 2. Department of Oral Pathology and Medicine, Okayama University Graduate School of Medicine, Dentistry and Pharmaceutical Sciences, Okayama, Japan \\ 3. Department of Oral and Maxillofacial Surgery, Shimane University Faculty of Medicine, Shimane, Japan \\ $\triangle$ Corresponding author: Shintaro Sukegawa, DDS, PhD, Consultant Surgeon, Division of Oral and Maxillofacial Surgery, Kagawa Prefectural Central \\ Hospital, 1-2-1 Asahi-cho, Takamatsu, Kagawa 760-8557, Japan. Tel: +81 87811 3333; Fax: +81 878358363 ; Email address: gouwan19@gmail.com, \\ s-sukegawa@chp-kagawa.jp \\ (C) Ivyspring International Publisher. This is an open access article distributed under the terms of the Creative Commons Attribution (CC BY-NC) license \\ (https://creativecommons.org/licenses/by-nc/4.0/). See http://ivyspring.com/terms for full terms and conditions.
}

Received: 2018.06.19; Accepted: 2018.10.18; Published: 2019.01.01

\begin{abstract}
Purpose: We aimed to document the clinical usefulness of uncalcined and unsintered hydroxyapatite (u-HA) particles and poly-L-lactide (PLLA) composite materials and their advantageous properties.

Methods: Between April 2016 and March 2018, five patients required anterior maxillary alveolar ridge augmentation using fixation with u-HA/PLLA screws for an onlay block bone graft harvested from the mandibular ramus at our institute. Bone biopsies were obtained from the dental implantation site following bone healing for histomorphometric and immunohistochemical (IHC) measurements.

Results: Many stromal cells were positive for Osterix, RUNX2, and SOX9 but were negative for CD68. On cell counting, based on IHC staining for Osterix, RUNX2, SOX9 and CD68 from peripheral u-HA/PLLA screw or bone areas, both areas consistently showed no significant difference in terms of Osterix, RUNX2, and SOX9. Hematoxylin-eosin staining revealed direct bone connection to the biomaterials, and no inflammatory cells infiltrated the areas surrounding the bone or artificial material. Area between the bone and u-HA/PLLA screw was seamless with no boundary. Round small cells and immature fibroblasts were noted. The new bone showed the presence of bone lamellae, normal osteocytes, and osteoblasts.

Conclusion: The u-HA/PLLA materials showed excellent biodegradability and bioactive osteoconductivity. In addition, this material induced no apparent inflammatory or foreign body reactions following implantation, and it directly bonded to the human bone. Therefore, this u-HA/PLLA material seems ideal and most suitable for use as a substitute for osteosynthesis.
\end{abstract}

Key words: Poly-L-lactide; uncalcined and unsintered hydroxyapatite; biodegradability; osteoconductivity; bone regeneration

\section{Introduction}

For internal fixation, bioresorbable osteosynthesis devices made of synthetic polymers have been developed and used as substitutes for metal osteosynthesis material. Osteosynthesis with absorbable material is an established and widely used treatment for maxillofacial conditions such as 
fractures [1,2], orthognathic surgery [3], bone augmentation [4], and reconstruction due to maxillofacial cysts and tumors [5,6]. Bioresorbable materials have certain advantages over metal osteosynthesis devices. First, device removal following bone healing is not required, as in the case of metal devices. Second, bioresorbable device use prevents adverse events usually caused by metallic materials such as thermal sensitivity [7], plate migration [8], and interference with diagnostic imaging [6]. These advantages support the potential of resorbable bone fixation devices in greatly contributing to development of future clinical treatments.

Currently, there are many commercially available osteosynthesis absorbable materials such as Super FIXSORB MX ${ }^{\circledR}$ (TEIJIN MEDICAL TECHNOLOGIES Co., Ltd. Osaka, Japan), i.e., OSTEOTRANS MX. These bioactive and bioresorbable devices are made from composites of uncalcined and unsintered hydroxyapatite (u-HA) particles and poly-L-lactide (PLLA) and are produced by a unique forging process incorporating compression molding and machining. Some advantageous properties of this composite material include its bone conduction and bone bonding capabilities, total long-term replacement of the bone, and desired mechanical properties, including ductility of a polymer and stiffness of human cortical bone $[9,10]$. Thus, although HA/PLLA material is a bioactive and bioresorbable material, it has stable retention characteristics in human bone tissue. Reportedly, the period until human bone healing and maturation in the maxillofacial area was approximately 6 months.[11] However, the characteristics of this device, particularly its histological features at the short-term to bone healing, are unclear. This is crucial while considering the bone healing period in the craniomaxillofacial area. Herein,
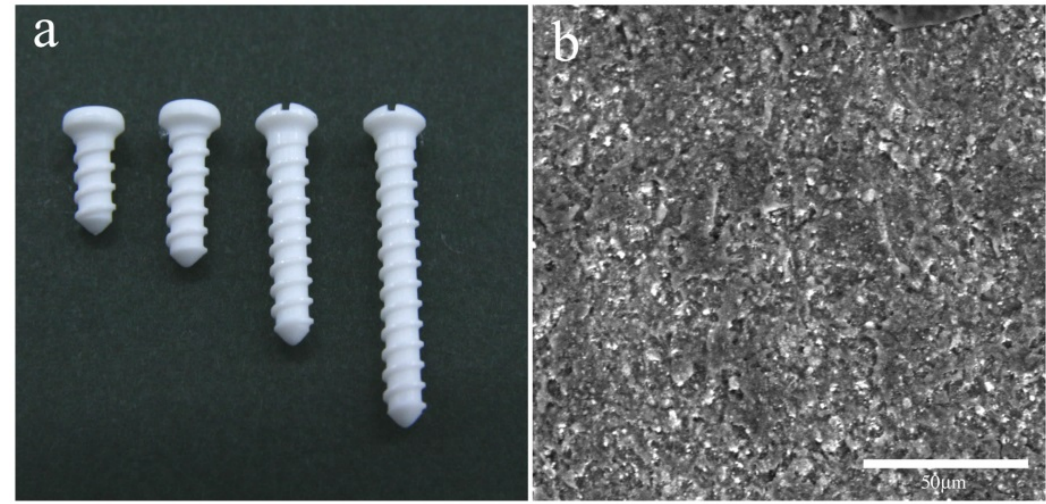

Figure 1. (A) Screws FIXSORB MX® ${ }^{\circledR}$ (TEIJIN MEDICAL TECHNOLOGIES Co., Ltd. Osaka, Japan) comprising forged composites of unsintered u-HA/PLLA. (B) SEM image of this material shows uniform dispersion of the u-HA fine particles on the surface. we document the clinical usefulness of u-HA/PLLA composites devices and their short-term advantages. We believe our study results will be useful to surgeons who select this device as a preoperative dental implant for bone augmentation.

\section{Methods}

\section{Materials}

In this study, the screw FIXSORB MX® (TEIJIN MEDICAL TECHNOLOGIES Co., Ltd. Osaka, Japan) comprising forged composites of unsintered u-HA/PLLA (containing 30 weight fractions of raw uncalcined, unsintered u-HA particles in composites) was used. The rods used had the following characteristics: diameter, $2.0 \mathrm{~mm}$; length, 6-12 mm; u-HA particle size, $0.2-20 \mu \mathrm{m}$ (average, 3-5 $\mu \mathrm{m}$ ); $\mathrm{Ca} / \mathrm{P}$ $=1.69$ (mol, ratio); and $\mathrm{CO}_{3}{ }^{2-}=3.8(\mathrm{~mol} \%)$ [10] (Figure $1)$.

\section{Subjects}

Between April 2016 and March 2018, at the Division of Oral and Maxillofacial Surgery, Kagawa Prefectural Central Hospital (Kagawa, Japan), five patients (two males and three females; age range, 29-76 years) required anterior maxillary alveolar ridge augmentation prior to dental implant placements with an onlay block graft harvested from the mandibular ramus (residual bone width, $<4 \mathrm{~mm}$ ).

\section{Surgical Bone Augmentation Procedure}

The donor and receptor sites were infiltrated with local anesthetic with patients under conscious intravenous sedation or general anesthesia, as appropriate. At the receptor site, a vertical incision was created using a scalpel in the vestibular gingival mucosa, mesial and distal to the bone defect. Following this, full-thickness flaps were elevated to facilitate satisfactory exposure of the recipient site. The remaining alveolar crest width was measured to determine the required bone collection volume. After preparing the recipient site, the cortical block bone was harvested from the mandibular ascending ramus. An oblique sagittal incision was made distal to the third molar equivalent part following the direction of the ramus. A vertical releasing incision was distally created in the mandibular ramus area. After reflection of buccal flaps, osteotomies were performed via piezosurgery to outline the dimensions of the bone block. On completion of osteotomy, a small bone chisel was placed along the sagittal cut and the lateral block of bone was 
greenstick fractured off. Then, the block autograft was fixed to the recipient site with one or two u-HA/PLLA screws. The sharp edges of the bone blocks were removed with large diamond burs. After the graft adapted to the site, an incision via the periosteum at the base of the flap facilitated the tissue to completely cover the graft without tension. Further, the recipient and donor sites were sutured (Surgisorb 4-0; Nitcho Corporation, Tokyo, Japan) without a barrier membrane.

Following 6-month bone regenerative healing process and prior to dental implantation, computerized tomography was performed to plan the dental implant surgery correctly and simultaneously and to confirm the position of the u-HA/PLLA screw. At implantation, biopsies were retrieved using a 2.0-mm diameter trephine burr (ACE Surgical Supply Company, Inc., Brockton, MA, USA) from the planned implant site, which had been decided during the bone augmentation operation, followed by placement of dental implants. All procedures were performed by an expert surgeon (S.S) at a single institution. This study was approved by the Ethics Committee of the Kagawa Prefectural Central Hospital (Approval No. 736).

\section{Preparation for Histological Evaluation}

All biopsies were immediately fixed in $4 \%$ paraformaldehyde for 12 hours and then decalcified in $10 \%$ ethylenediaminetetraacetic acid at $4^{\circ} \mathrm{C}$ for 14 days. The tissue was routinely embedded in paraffin and five thick serial sections were prepared. The sections were used for hematoxylin-eosin (HE) staining and immunohistochemistry (IHC).

\section{Immunohistochemistry}

IHC was performed using the antibodies detailed in Table 1. Following antigen retrieval, the sections were treated with $10 \%$ normal serum for 30 min, followed by incubation with primary antibodies at $4^{\circ} \mathrm{C}$ overnight. The immunoreactive site was identified using the avidin-biotin complex method (Vector Laboratories, Burlingame, CA, USA).

Table 1. List of antibodies used in this study.

\begin{tabular}{lllll}
\hline $\begin{array}{l}\text { Primary } \\
\text { antibody }\end{array}$ & $\begin{array}{l}\text { Immunized } \\
\text { animal }\end{array}$ & Antigen retrieval & Dilution Supplier \\
\hline Osterix & Rabbit & $\begin{array}{l}\text { Heated in } 0.01 \mathrm{~mol} / \mathrm{L} \text { citrate } \\
\text { buffer for } 1 \mathrm{~min}\end{array}$ & $1: 100$ & abcam \\
RUNX 2 & Mouse & $\begin{array}{l}\text { Heated in } 0.01 \mathrm{~mol} / \mathrm{L} \text { citrate } \\
\text { buffer for } 1 \mathrm{~min}\end{array}$ & $1: 500$ & abcam \\
SOX9 & Rabbit & $\begin{array}{l}\text { Heated in } 0.01 \mathrm{~mol} / \mathrm{L} \text { citrate } \\
\text { buffer for } 1 \mathrm{~min}\end{array}$ & $1: 100$ & abcam \\
CD68 & Mouse & $\begin{array}{l}\text { Heated in } 0.01 \mathrm{~mol} / \mathrm{L} \text { citrate } \\
\text { buffer for } 1 \mathrm{~min}\end{array}$ & $1: 100$ & DAKO \\
\hline
\end{tabular}

\section{Histomorphometry}

To compare the effect of the u-HA/PLLA screw among the sections from bone augmented areas, each specimen was divided into two areas: peripheral bone area (tissue away from the u-HA/PLLA screw) and peripheral u-HA/PLLA screw area (tissue connecting to the u-HA/PLLA screw). Cell counting was performed in each area. Histological analysis and characterization of the resected specimen were performed based on this area division. Following counterstaining with hematoxylin, the sections were microscopically examined at $\times 400$ magnification. Five areas were randomly selected in each sample, 100 cells were counted in each area, and the percentage of positive cells was calculated and compared between the groups. All counting was performed thrice in each specimen by a pathologist specialized in bone evaluation.

\section{Statistical Analysis}

Data were recorded and entered into an electronic database during the course of the evaluation using Microsoft Excel (Microsoft, Inc., Redmond, WA, USA). Means and standard deviations are used for normal data distributions. Statistical differences were calculated and analyzed using the Mann-Whitney $U$-test. The database was transferred to JMP version 11.2 for Macintosh computers (SAS Institute, Inc., Cary, NC, USA) for statistical analysis. $P<0.05$ was considered statistically significant.

\section{Results}

\section{Clinical Evaluation}

Six months following anterior maxillary alveolar bone regenerative augmentation, all patients had sufficient bone volume for placement of dental implants. The implants were left in place for an average of 5-6 months prior to preparation of the definitive prosthesis and loading. Remarkably, we observed no implant failures. Five biopsies were retrieved from the augmented area with the $\mathrm{u}-\mathrm{HA} / \mathrm{PLLA}$ screw at the beginning of implantation using a trephine burr.

\section{Histopathological Examination}

In all five cases, the excisional material was examined using HE staining. On loupe image, the bone component was mixed with the u-HA/PLLA screw (Figure 2A). On high power field, the bone directly connected to the biomaterials, and no inflammatory cells infiltrated the space between the bone and artificial material. The boundary between the bone and u-HA/PLLA screw was seamless. Few inflammatory cells were noted at the peripheral areas 
of the bone or u-HA/PLLA screw, with round small cells and immature fibroblasts. The new bone showed bone lamellae, normal osteocytes and osteoblasts and had the characteristics of a normal bone (Figure 2B-E).

\section{Immunohistochemical Examination}

Many stromal cells were positive for Osterix, RUNX2, and SOX9. These cells were small and round in shape and were negative for CD68. Further, there were very few CD68 single positive cells in the same area (Figure 3A-H).
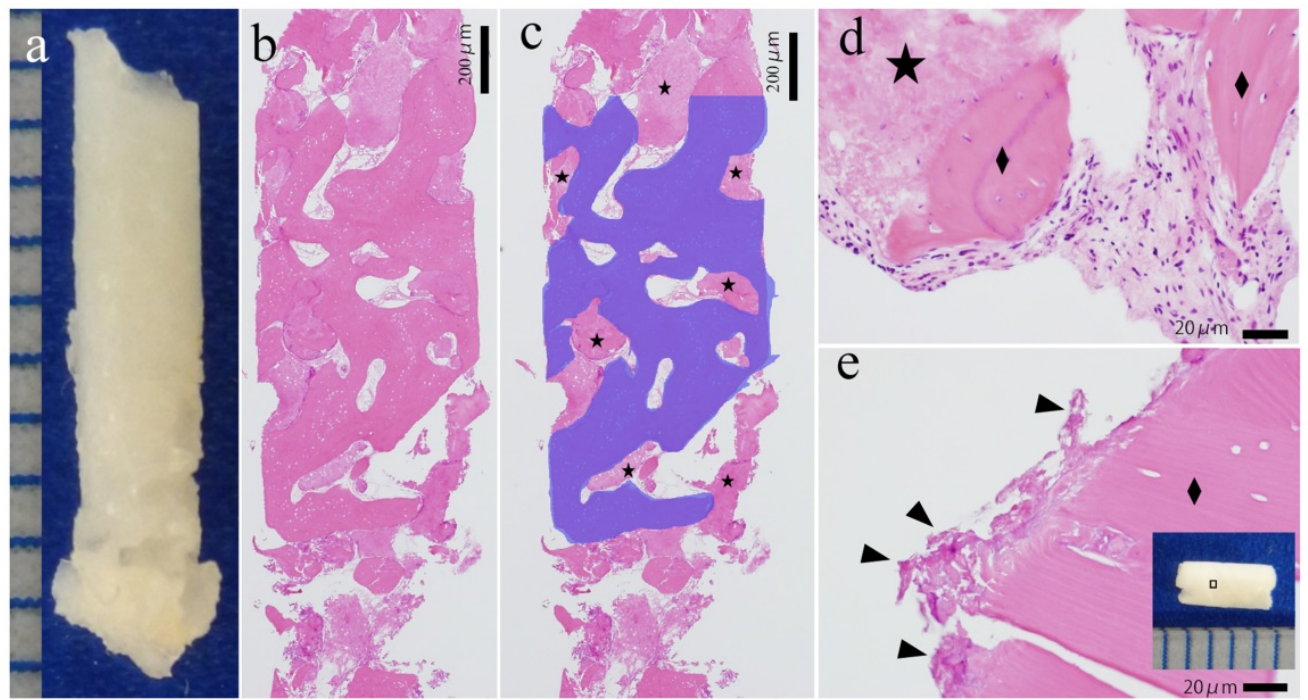

Figure 2. Histological evaluation of the excisional material. (A) Excisional material. (B, C) Loupe image of HE staining. The blue area is the bone tissue. The star ( $\star$ ) indicates the u-HA/PLLA material. Bone and u-HA/PLLA material existed in a mixed form in excisional material. Bar: $200 \mu \mathrm{m}$. (D, E) High power image of HE staining. (D) The u-HA/PLLA material contacted the new bone, and there was no border or inflammation. The diamond indicates $(\diamond)$ the bone. (E) The remaining material (arrowhead) directly connected to the peripheral bone. The dotted line indicates border bone and the u-HA/PLLA material. Bar: $20 \mu \mathrm{m}$.

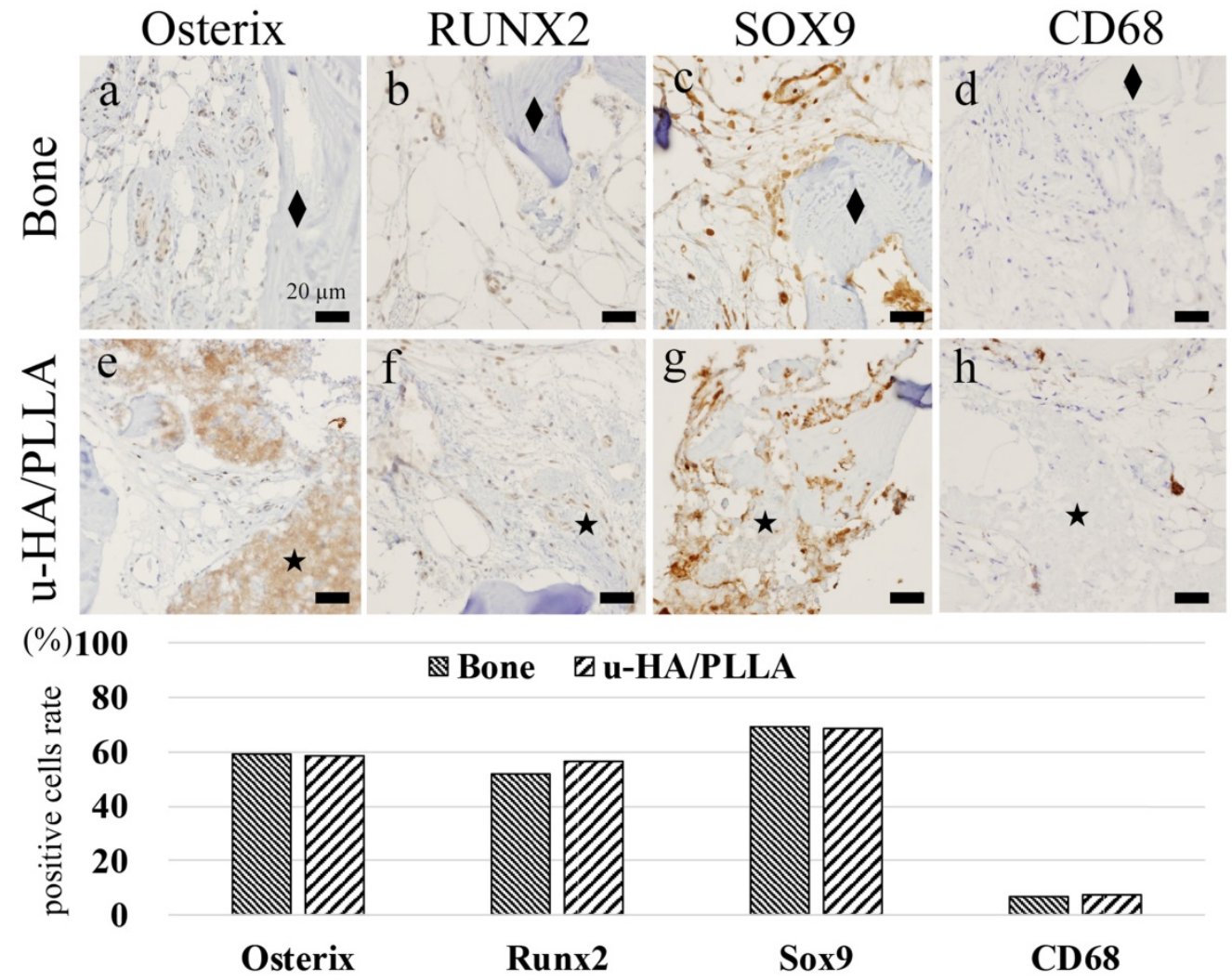

Figure 3. Analysis of IHC. (A-D) Peripheral bone area: (A) Osterix, (B) RUNX2, (C) SOX9, and (D) CD68. The diamond indicates $(\bullet)$ the bone, and the star ( $\star$ ) indicates the u-HA/PLLA material. Many Osterix-, RUNX2-, and SOX9-positive cells were observed in the connective tissue. CD68-positive cells were not identified. Bar: 20 m. (E-H) Peripheral u-HA/PLLA area: (E) Osterix, (F) RUNX2, (G) SOX9, and (H) CD68. The star ( $\star$ ) indicates the u-HA/PLLA material. Osterix-, RUNX2-and SOX9-positive cells were observed in the peripheral connective tissue. This distribution was similar to that in the peripheral bone tissue. CD68-positive cells were not observed. Bar: $20 \mu m$. (I) Comparison of positive cell ratios between the bone and u-HA/PLLA material. Both ratios are similar, with no significant difference. 


\section{Cell Counting Analysis of IHC Staining}

Results of cell counting based on IHC staining for Osterix, RUNX2, SOX9, and CD68 according to the area divisions of peripheral u-HA/PLLA screw or bone are shown in the histograms (Figure 3I). Both areas consistently showed no significant difference in terms of Osterix, RUNX2, and SOX9.

\section{Discussion}

Research on resorbable materials has been well described in the literature since the 1970s [12] and has substantially developed to date. Various polymers have been investigated, including polyglycolic acid (PGA), polylactic acid, PLLA, and poly-d-lactate [13], a copolymer of PGA/PLLA $[13,14]$, and u-HA/PLLA $[15,16]$. The literature well documents that resorbable materials offer many advantages for osteosynthesis with metal devices. Because removal of osteosynthesis material is not necessary, complications associated with screw removal, such as risk of damage to the healed bone and fracture of the material [18], can be avoided. In addition, the elasticity of these bioresorbable materials is close to that of the human bone, thereby preventing stress-shielding atrophy and weakening of the fixed bone caused by rigid metallic fixation [18- 20]. During bone healing, the bioresorbable material gradually deteriorates and can resist physiological stress for achieving bone healing in the end. As a result, a major advantage is that stress shielding is avoided in bioresorbable materials.

However, bone healing around bioresorbable materials has some problems. Raghoebar et al. [21] reported a clinical study on the application of PGA/PLLA biodegradable screws to fix bone grafts in a human model. They reported that giant cells are abundantly found around bioresorbable screws compared with titanium and resorbable screws, although there was no indication of wound healing failure around the bioresorbable materials and no severe inflammatory response to PDLLA histologically. Only partial bone healing was reported around the remaining bioresorbable screw. Remarkably, the u-HA/PLLA bioresorbable screws completely overcame this weakness. Inflammatory cells minimally infiltrated the area around the u-HA/PLLA screws in our histological analysis. Furthermore, immunohistochemical analysis revealed that very few CD68 positive cells were peripherally observed around the u-HA/PLLA bioresorbable screws, indicating that inflammation was suppressed because CD68 is a macrophage marker. In addition, this showed that the u-HA/PLLA material is not only bioresorbable but also has good biocompatibility. This result is consistent with that reported in a previous animal study [22]. In this previous study, histological evaluation of specimens from a fracture treatment model in a white rabbit using the same materials between 4 and 52 weeks showed neither macrophages nor inflammatory giant cells over time. It was inferred that the effect of the combination of $\mathrm{u}-\mathrm{HA}$ and PLLA resulted in stable hydrolysis to degradation of PLLA from immediately following transplantation in vivo. Furthermore, because the HA particles of this material were mainly formed due to the chemical dissolution process, they did not induce inflammatory and foreign body reactions. Furthermore, regarding the long-term course, we previously reported about the successful use of this plate over 5 years following implantation [23], followed by complete decomposes in the human body [24]. Despite having the same initial chemical composition as that following sintering, the in vivo behavior of the sintered or unsintered HA/PLLA material was found to be different. This may be due to a change in their solubility as a result of the evolution of their surface morphology with sintering temperature. Therefore, our study indicated that (1) the fine particle structure of u-HA/PLLA surface is a form difficult to be recognized by macrophages and inflammatory cells, (2) it does not cause inflammation, and (3) the u-HA/PLLA material can sufficiently exhibit its original osteoinductivity.

Normally, the bioresorbable materials are gradually replaced with bone in the absorbed part, and bone healing progresses [4]. Therefore, there is no continuous seamless autogenous bone and bioresorbable material. However, in our study, we did not observe a border between the bone and u-HA/PLLA screws, indicating that the material directly bonded with the human bone. Yasunaga et al. [25] reported that no direct contact only between the PLLA material and bone cortex was detectable on histological examination at any post-implantation period in a rabbit study. On the other hand, regenerative bone tissue was seen to be directly connected to the u-HA/PLLA material without any intervening tissue such as fibrous and granulation tissues. They concluded that these differences in terms of bonding strength between the u-HA/PLLA and PLLA-only materials were due to inclusion of u-HA particles in the material. Our research is significantly valuable in that it showed direct bonding of this material to human cortical bone at a relatively early period.

Osterix-, RUNX2-, and SOX9-positive cells are considered preosteoblasts. Therefore, we evaluated the presence of RUNX2 and SOX9, essential transcription factors for osteogenitor cells in the sequence of events leading to osteoblast 
differentiation [26], and Osterix, an essential marker of bone calcification pathways [27]. In our study, RUNX2 and SOX9 were shown to localize to cells of osteogenic or chondrogenic lineage in human tissues. The expression of these three antibodies indicates that the cells are preosteoblasts because these markers are expressed during the early stages of osteoblast differentiation [28]. In our specimens, RUNX2- and SOX9-positive cells were mainly localized around the $\mathrm{u}-\mathrm{HA} / \mathrm{PLLA}$ material and in newly formed bone tissues. Moreover, Osterix-positive cells were present in preosteoblasts and newly formed bone tissue. Many preosteoblasts exist in the stroma, and this microenvironment can potentially form bone tissue. We confirmed that the preosteoblasts infiltrated in the connective tissues around the u-HA/PLLA material are osteogenic and subject to the osteogenic differentiation pathway around this material. The IHC results indicate that both areas (new bone and peripheral tissue of materials) are similar. The peripheral tissue of these materials can potentially replace new bone positively in the same manner on the side of the new bone.

The most ideal osteosynthesis material may be a bone screw made of autologous cortical bone that does not require removal, has osteointegrative properties, and lacks a foreign body reaction. However, there is a significant limit to this material. Application of this technique has some limitations in the clinical setting. First, the donor site is limited, limiting the number of bone screws that can be made from this site. Second, a risk of fracture and damage at the donor site are possible. Lastly, a dedicated screw-making tool is necessary, which is difficult to inexpensively use for this method to be equally performed at all facilities [29]. The bioresorbability and osteoconductive bioactivity of $\mathrm{u}-\mathrm{HA} / \mathrm{PLLA}$ are distinct advantages for maxillofacial bone regeneration. Therefore, this material is substantially different from bioactive ceramics, which have poor flexibility due to their insufficient bone substitution ability, and is most suitable for use as a substitute for osteosynthesis.

Most studies concerning histological examination of bioresorbable materials have been performed in animals $[16,25]$. Unlike metal osteosynthesis plate systems, because bioresorbable materials are absorbable without requiring reoperation for their removal, their examination in the human body is ethically difficult. Previous studies on bioresorbable materials requiring removal owing to complications were only conducted for clinical research in a small number of cases, and their biological conditions varied $[2,14,30]$. To the best of our knowledge, the preliminary results obtained herein could shed light on the first attempt to clarify biodegradability and bioactive osteoconductivity as histological evaluations of u-HA / PLLA bioresorbable materials in human maxillofacial bones; this is the first description of this interesting and significant novel concept.

\section{Conclusion}

The u-HA/PLLA material showed excellent biodegradability and bioactive osteoconductivity in this study. In addition, this material induced no apparent inflammatory or foreign body reactions following implantation, and it directly bonded to the human bone. Therefore, $\mathrm{u}$-HA/PLLA material is ideal and most suitable for use as a substitute for osteosynthesis. Furthermore, regarding CD68 positive cells, their appearance indicates poor bone remodeling around the materials. This environment possibly promotes bone growth. This histological characteristic provides long-term stability of the bone tissue for the u-HA/PLLA material.

\section{Acknowledgements}

This study was jointly funded by the JSPS KAKENHI Grant Numbers 26462783, 16K20577.

\section{Ethical Approval}

This study was approved by the Ethics Committee of the Kagawa Prefectural Central Hospital (Approval No. 736).

\section{Competing Interests}

The authors have declared that no competing interest exists.

\section{References}

[1] Sukegawa S, Kanno T, Nagano D, Shibata A, Sukegawa-Takahashi Y, Furuki $\mathrm{Y}$. The clinical feasibility of newly developed thin flat-type bioresorbable osteosynthesis devices for the internal fixation of zygomatic fractures. J Craniofac Surg. 2016; 27: 2124-9.

[2] Sukegawa S, Kanno T, Katase N, Shibata A, Takahashi Y, Furuki Y. Clinical evaluation of an unsintered hydroxyapatite/poly-l-lactide osteoconductive composite device for the internal fixation of maxillofacial fractures. J Craniofac Surg. 2016; 27: 1391-7.

[3] Paeng JY, Hong J, Kim CS, Kim MJ. Comparative study of skeletal stability between bicortical resorbable and titanium screw fixation after sagittal split ramus osteotomy for mandibular prognathism. J Cranio-Maxillofacial Surg. 2012; 40: 660-4

[4] Quereshy FA, Dhaliwal HS, El SA, Horan MP, Dhaliwal SS. Resorbable screw fixation for cortical onlay bone grafting: a pilot study with preliminary results. J Oral Maxillofac Surg. 2010; 68: 2497-502.

[5] Sukegawa S, Kanno T, Shibata A, et al. Intraoperative navigation-assisted accurate bone lid surgery to remove a mandibular lesion: A case report. Oral Maxillofac Surg Cases. 2017; 3: 15-9.

[6] Sukegawa S, Kanno T, Shibata A, et al. Use of the bioactive resorbable plate system for zygoma and zygomatic arch replacement and fixation with modified Crockett's method for maxillectomy: A technical note. Mol Clin Oncol. 2017; 7: 47-50

[7] Manson PN, Hoopes JE, Su CT. Structural pillars of the facial skeleton: an approach to the management of Le Fort fractures. Plast Reconstr Surg. 1980; 66: 54-62.

[8] Fearon JA, Munro IR, Bruce DA. Observations on the use of rigid fixation for craniofacial deformities in infants and young children. Plast Reconstr Surg. 1995; 95: 634-7; discussion 638. 
[9] Shikinami Y, Matsusue Y, Nakamura T. The complete process of bioresorption and bone replacement using devices made of forged composites of raw hydroxyapatite particles/poly 1-lactide (F-u-HA/PLLA). Biomaterials. 2005; 26: 5542-51.

[10] Shikinami Y, Okuno M. Bioresorbable devices made of forged composites of hydroxyapatite (HA) particles and poly-L-lactide (PLLA): Part I. Basic characteristics. Biomaterials. 1999; 20: 859-77.

[11] RE Marx. Bone and bone graft healing. Oral Maxillofac Surg Clin North Am. 2007; 19: 455-66.

[12] Cutright DE, Hunsuck EE. The repair of fractures of the orbital floor using biodegradable polylactic acid. Oral Surg Oral Med Oral Pathol. 1972; 33: 28-34.

[13] Wimpenny I, Lahteenkorva K, Suokas E, Ashammakhi N, Yang Y. Improvement and characterization of the adhesion of electrospun PLDLA nanofibers on PLDLA-based 3D object substrates for orthopedic application. J Biomater Sci Polym Ed. 2012; 23: 1863-77.

[14] Sukegawa S, Kanno T, Matsumoto K, Sukegawa-Takahashi Y, Masui M, Furuki Y. Complications of a poly-L-lactic acid and polyglycolic acid osteosynthesis device for internal fixation in maxillofacial surgery. Odontology. 2018; 7: 1-9.

[15] Sukegawa S, Kanno T, Shibata A, Takahashi Y, Furuki Y. Use of templates and self-tapping metal screws for temporary fixation of a resorbable plate system. Ann Maxillofac Surg. 2015; 5: 231-3.

[16] Hasegawa S, Ishii S, Tamura J, et al. A 5-7 year in vivo study of high-strength hydroxyapatite/poly(L-lactide) composite rods for the internal fixation of bone fractures. Biomaterials. 2006; 27: 1327-32.

[17] Furukawa T, Matsusue Y, Yasunaga T, et al. Histomorphometric study on high-strength hydroxyapatite/poly(L-lactide) composite rods for internal fixation of bone fractures. J Biomed Mater Res. 2000; 50: 410-9.

[18] Quereshy FA, Dhaliwal HS, El SA, Horan MP, Dhaliwal SS. Resorbable screw fixation for cortical onlay bone grafting: a pilot study with preliminary results. J Oral Maxillofac Surg. 2010; 68: 2497-502.

[19] Pietrzak WS, Verstynen ML, Sarver DR. Bioabsorbable fixation devices: status for the craniomaxillofacial surgeon. J Craniofac Surg. 1997; 8: 92-6.

[20] Devin JE, Attawia MA, Laurencin CT. Three-dimensional degradable porous polymer-ceramic matrices for use in bone repair. J Biomater Sci Polym Ed. 1996; 7: 661-9.

[21] Raghoebar GM, Liem RSB, Bos RRM, van der Wal JE, Vissink A. Resorbable screws for fixation of autologous bone grafts. Clin Oral Implants Res. 2006; 17: 288-93.

[22] Furukawa T, Matsusue Y, Yasunaga T, Shikinami Y, Okuno M, Nakamura T. Biodegradation behavior of ultra-high-strength hydroxyapatite/poly (L-lactide) composite rods for internal fixation of bone fractures. Biomaterials. 2000; 21: 889-98.

[23] Sukegawa S, Kanno T, Kawai H, Shibata A, Matsumoto K, Takahashi Y, et al. Surgical treatment and dental implant rehabilitation after the resection of an osseous dysplasia. J Hard Tissue Biol. 2016; 25: 437-41.

[24] Sukegawa S, Kanno T, Kawai H, Shibata A, Takahashi Y, Furuki Y. Long-term bioresorption of bone fixation devices made from composites of unsintered hydroxyapatite particles and poly-L-lactide. J Hard Tissue Biol. 2015; 24: 219-24.

[25] Yasunaga T, Matsusue Y, Furukawa T, Shikinami Y, Okuno M, Nakamura T. Bonding behavior of ultrahigh strength unsintered hydroxyapatite particles/poly(L-lactide) composites to surface of tibial cortex in rabbits. J Biomed Mater Res. 1999; 47: 412-9.

[26] Rodan GA, Harada S. The missing bone Cell 1997: 89: 677-80.

[27] Nakashima K, Zhou X, Kunkel G, et al. The novel zinc finger-containing transcription factor osterix is required for osteoblast differentiation and bone formation. Cell. 2002; 108: 17-29.

[28] Rutkovskiy A, Stensløkken KO, Vaage IJ. Osteoblast differentiation at a glance. Med Sci Monit Basic Res. 2016; 22: 95-106.

[29] Kumahashi N, Kuwata S, Imade S, Kono M, Takuwa H, Uchio Y. Fixation of osteochondral fractures of the patella using autologous bone screws when reconstructing the medial patellofemoral ligament after recurrent patellar dislocation: report of two cases. J Orthop Sci. 2014; 19: 359-64.

[30] Kanno T, Sukegawa S, Furuki Y, Nariai Y, Sekine J. Overview of innovative advances in bioresorbable plate systems for oral and maxillofacial surgery. Jpn Dent Sci Rev. 2018; 54: 127-138. 